\title{
Method of the Year 2020: spatially resolved transcriptomics
}

\author{
Spatially resolved transcriptomics methods are changing the way we understand complex tissues.
}

U nderstanding the organization of cells and tissues and how this organization influences function is a fundamental pursuit in life sciences research. Over the years, technological advances have shed much light on these aspects of biology. Sequencing approaches have allowed us to learn about cell types and their heterogeneity in complex tissues, and methodological advances in microscopy, including super-resolution and single-molecule imaging, have transformed our understanding of cell and tissue structure and function. Our Method of the Year, spatially resolved transcriptomics, highlights how these technologies have matured and expanded to give biologists exceptional views of the biology of single cells while retaining information on spatial context.

Although we have covered single-cell technologies before - including in 2013, when we featured single-cell RNA and DNA sequencing, and even just last year, with our selection of single-cell multimodal omics - our decision this year to highlight spatially resolved transcriptomics was unanimous because, unlike the methods highlighted previously, these methods are able to elucidate single-cell heterogeneity and define cell types while also retaining spatial information. This maintenance of spatial context is crucial for understanding key aspects of cell biology, developmental biology, neurobiology, tumor biology and more, as specialized cell types and their specific organization are crucially tied to biological activity and remain poorly explored on the scale of whole tissues and organisms. As such, large consortia such as the Human Cell Atlas and Brain Initiative Cell Census Network (BICCN) are making use of spatially resolved transcriptomics technology, with the ultimate goal of generating complete maps of large and complex tissues like the human brain.

Broadly speaking, spatially resolved transcriptomics studies are carried out one of two ways. Transcriptomes can be read out by microscopy through in situ sequencing or multiplexed fluorescence in situ hybridization (FISH). Alternatively, RNA is captured in a way that retains spatial information while sequencing is done using ex situ RNA-seq. These approaches are often complementary and differ in their target coverage, spatial resolution and throughput; like all methods, they must be appropriately matched to their biological questions.

$$
\text { A Comment from Xiaowei }
$$

Zhuang provides an in-depth look at imaging-based methods for spatially resolving transcriptomes. These methods historically have offered the highest spatial resolution, providing access to subcellular information, but have been limited in terms of sequence coverage and overall throughput. The Comment highlights FISH-based approaches and how they have been pushed to reach higher numbers of targets or to cover larger numbers of cells. It also covers in situ sequencing approaches and their capabilities and challenges, such as target density and throughput speed, and how these challenges are being met. The piece also discusses how spatially resolved transcriptomics have enabled subcellular transcriptome mapping and how this relates to genome organization.

Another Comment, from Joakim Lundeberg and colleagues, describes methods that marry spatial mapping and single-cell RNA-seq (scRNA-seq). In these approaches, cellular RNAs are barcoded in a grid-like fashion on the basis of their position in a tissue slice before homogenization and scRNA-seq. These methods offer unbiased capture of the transcriptomic landscape with relative high throughput, but do not yet offer subcellular resolution. This piece covers recent efforts toward improving resolution, as well as applications to mapping organs by spatial gene expression and transcriptional changes of healthy and diseased tissues. Lundeberg and colleagues anticipate multimodal spatial profiling that enables parallel measurements of the transcriptome, genome and proteome.

A third Comment, from Hongkui Zeng and colleagues at the BICCN project, explores why these technologies are so important for understanding complex tissues and the progress they and others have made in mapping cellular organization of the brain. The researchers also share challenges they've faced, such as mapping reads back to their cell of origin and issues of scalability.

And finally, our Technology Feature brings together many voices from the field to highlight why researchers are so excited about these approaches, how they are being applied to challenging biological questions, and what the future might look like as technology develops further.

Although these methods are becoming well-established and are seeing use far beyond the labs in which they originated, we anticipate future developments will further drive these approaches into the mainstream. In terms of imaging-based approaches, we expect that advances in large-field-of-view microscopes that offer high resolution will increase throughput. We also think that improved sample preparation methods like tissue clearing and expansion microscopy will help tackle challenges like autofluorescence and crowding. For ex situ sequencing based approaches, we expect that improvements in sequencing technology will improve sensitivity and coverage and that advances in barcoding platforms (such as the use of microbeads and microfluidics) and combinations of barcoding strategies will lead to improved data quality and subcellular resolution. We also foresee software tools for automated processing of the data and for dealing with huge datasets proving important. Finally, we see multimodal applications as vital for increasing information content from tissues.

We invite you to explore this special issue. It also includes Methods to Watch, where we highlight technologies we think are poised to be important in the future of biological research.

Published online: 6 January 2021

https://doi.org/10.1038/s41592-020-01042-X 